\title{
Revealing the Iron Oxides Mineral Core in Ferritin due to the Variations in the $H$ and L Subunits
}

\author{
Surya Narayanan ${ }^{1}$, Emre Firlar ${ }^{1,2}$, Shayan Shafiee ${ }^{1}$, Kun $\mathrm{He}^{2}$, Reza Shahbazian-Yassar ${ }^{2}$, Tolou \\ Shokuhfar ${ }^{1,3}$ \\ 1. University of Illinois at Chicago, Department of Bioengineering, Chicago, IL, USA \\ 2. University of Illinois at Chicago, Department of Mechanical and Industrial Engineering, Chicago, IL, \\ USA \\ ${ }^{3 .}$ University of Illinois at Chicago, College of Dentistry, Chicago, IL, USA
}

Iron is an essential element involved in electron transfer processes in many biological reactions in the human body. Excess iron is stored and regulated in ferritin proteins during the biomineralization and demineralization processes. Cytosolic ferritin is $12 \mathrm{~nm}$ in diameter and composed of a 24-subunit protein shell containing Heavy $(\mathrm{H})$ and Light $(\mathrm{L})$ chains and a ferritin iron core approximately 8nm in diameter. The $\mathrm{H}$ subunits are responsible for iron detoxification while $\mathrm{L}$ subunits are responsible for iron storage. Ferritin $\mathrm{H}$ and $\mathrm{L}$ subunit ratios are unique to each organ within the human body. $\mathrm{H}$ rich ferritins are prominent in organs such as human heart and brain while the L rich ferritins are primarily observed in storage organs such as the liver and spleen [1].

Understanding the mechanisms involved in the iron core formation as well the characterization of the iron core structure in ferritin has been a subject of interest from many years. Evidence indicates the association of the iron oxide core composition in dysfunctional ferritins with many neurological and non-neurological diseases such as Alzheimer's disease, Parkinson's disease, Cardiomyopathy, and Hemochromatosis. Magnetite and maghemite were the most commonly observed iron oxides in dysfunctional ferritin [3]. Different studies suggest possible reasons for different iron core compositions, which include the rate of biomineralization [2] and demineralization processes [4] and the electron beam effect on the material, which could alter the core structure [5]. To explore the biomineralization and demineralization processes and further understand the formation of different iron oxides, the contribution of $\mathrm{H}$ and $\mathrm{L}$ subunits needs to be better understood.

In the present study, commercially available $\mathrm{H}$ rich and the $\mathrm{L}$ rich ferritins obtained from healthy human hearts and human spleens were compared. In order to prevent artifacts and electron beam damage, the characterization of the proteins was performed in the native liquid state of the protein by utilization of Graphene Liquid Cells (GLCs). The high electron conductivity of graphene and the ability of GLCs to encapsulate thin liquid samples [6] were implemented to obtain spatially resolved Electron Energy Loss Spectroscopy (EELS) to determine the oxidation states of iron. Selected Area Electron Diffraction (SAED) analyses of the crystal structures were obtained to identify different iron oxides.

The studies were carried out with controlled electron dosage at a low electron voltage of 80keV. From EELS data (Figure 1), the ratio of $\mathrm{Fe}^{+2} / \mathrm{Fe}^{+3}$ was 0.24 in human heart ferritin (HHF), indicating the presence of hematite $\left(\alpha-\mathrm{Fe}_{2} \mathrm{O}_{3}\right)$. However, the ratio of $\mathrm{Fe}^{+2} / \mathrm{Fe}^{+3}$ was 0.50 in human spleen ferritin (HSF), indicating the presence of magnetite $\left(\mathrm{Fe}^{+2} \mathrm{Fe}^{+3}{ }_{2} \mathrm{O}_{4}\right)$ as a major iron oxide in the $\mathrm{L}$ rich ferritins. To further characterize the iron oxides of the proteins, SAED (Figure 2) was performed, which indicated the presence of hematite with trace amounts of maghemite $\left(\gamma-\mathrm{Fe}_{2} \mathrm{O}_{3}\right)$, ferrihydrite $\left(\mathrm{Fe}^{+3}{ }_{2} \mathrm{O}_{3} \cdot \mathrm{O}_{.5} \mathrm{H}_{2} \mathrm{O}\right)$, and magnetite in HHF while the HSF showed the presence of maghemite and magnetite with trace amounts of hematite. 
In $\mathrm{HHF}$, detoxification of iron is given primary importance due to which the number of $\mathrm{Fe}^{+3}$ ions are higher compared to $\mathrm{Fe}^{+2}$ forming iron oxides with majority of them as Hematite. However, in HSF, the equal amounts of $\mathrm{Fe}^{+2}$ and $\mathrm{Fe}^{+3}$ indicate the presence of stable detoxified iron (III) oxide and Iron (II) species that can be utilized during the demineralization processes. As a long-term goal, the iron core composition of healthy ferritin will be compared to dysfunctional ferritin [7].

\section{References:}

[1] Massover, W.H., Micron 24 (1993), p. 389.

[2] Collingwood J.F. et al, Journal of Alzheimer's Disease 14 (2008), p.235.

[3] C.Quintana, J.M. Cowley, and C.Marhic, Journal of Structural Biology 147 (2004), p. 166.

[4] Galvez N. et al, Journal of American Chemical Society 130 (2008), p. 8062.

[5] Pan. Y.H. et al, Journal of Structural Biology 166 (2009), p. 22.

[6] Wang C. et al, Advanced Materials 26 (2014), p. 3410.

[7] The authors acknowledge funding from the National Science Foundation - CAREER award- Grant

No- DMR 1564950. Prof. Tolou Shokuhfar is acknowledged for her many useful discussions and contribution to this work. This work made use of instruments in the Electron Microscopy Service (Research Resources Center, UIC).
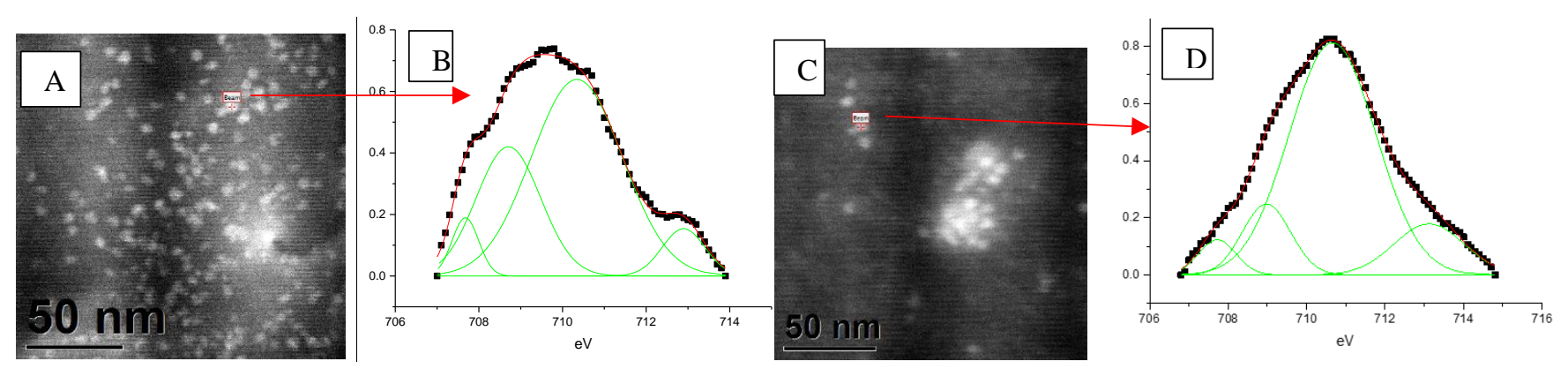

Figure 1. Shows the EELS Spectrum (Fig B) of HSF acquired from the area in Fig A, Fig D shows the EELS Spectrum acquired from HHF from the area as shown in Fig $\mathrm{C}$.
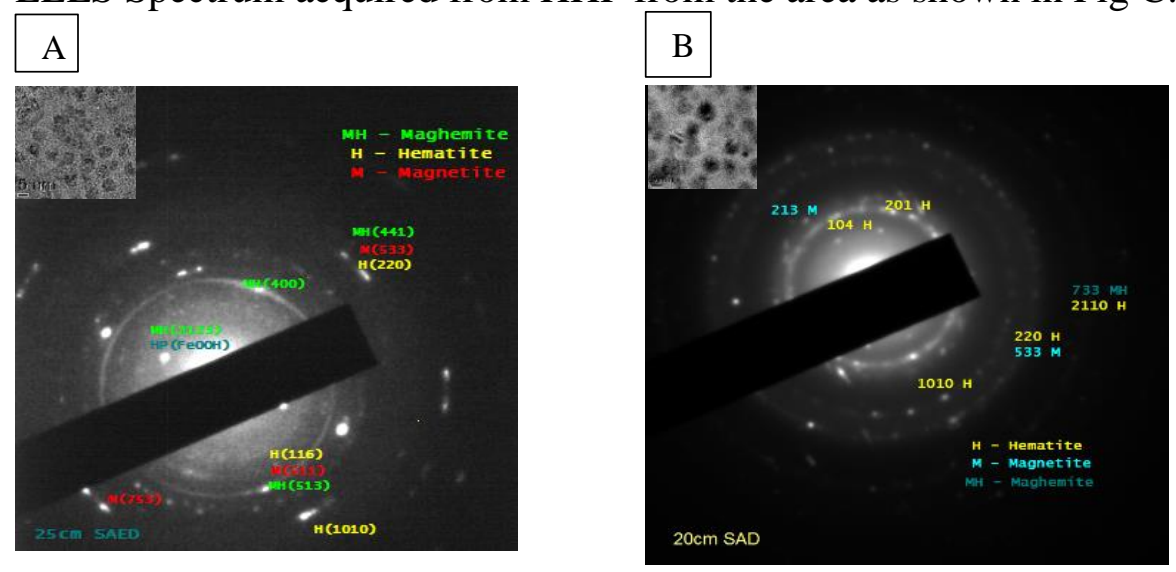

Figure 2. Shows the SAED of A) HSF and B) HHF acquired from the selected area of the sample. 